\title{
STRANGENESS AND PARTICLE FREEZE-OUT IN NUCLEAR COLLISIONS AT 14.6 GeV A
}

\author{
J. Letessier ${ }^{1}$, J. Rafelski ${ }^{1,2}$ and A. Tounsi ${ }^{1}$ \\ ${ }^{1}$ Laboratoire de Physique Théorique et Hautes Energies*, Paris \\ ${ }^{2}$ Department of Physics, University of Arizona, Tucson, AZ 85721
}

\begin{abstract}
We study the chemical conditions at freeze-out associated with the production of strange-particles in $\mathrm{Si}-\mathrm{Au}$ collisions at $14.6 \mathrm{GeV} \mathrm{A}$. We obtain freeze-out chemical potentials and temperature, and determine the entropy as well as the final particle abundance. We also consider in detail the alternative evolution scenarios involving the hadronic gas and the deconfined phase.
\end{abstract}

1. Introduction. In the past years a number of experiments at $14.6 \mathrm{GeV}$ A (AGS-BNL) and at $200 \mathrm{GeV}$ A (SPS-CERN) have shown that the strange particle abundance differs considerably in nuclear interactions from that in nucleon-nucleon collisions [1]. Qualitative agreement with the experiment within the ARC particle cascade models has been reached at the AGS energy [2], 3], without need for any novel mechanisms of strange particle production enhancement, but at CERN energies such agreement has not been obtained [ []] - moreover so far no microscopic model has emerged, capable to describe in all necessary detail the multistrange anti-baryon abundance seen at CERN in the central rapidity region. Furthermore one finds significant difference in the strangeness flow comparing $14.6 \mathrm{GeV}$ A with $200 \mathrm{GeV}$ A collisions [5].

The ARC results indicate transient presence of a clearly defined region of high density matter at central rapidity, seen in particular in the case of the $\mathrm{Au}-\mathrm{Au}$ collision simulations. This suggests that a local equilibrium thermal model (fireball model) may also do well in describing these collisions and in this work we will assume simply that all central rapidity strange particles originate from a central, thermally equilibrated fireball — our analysis will allow in principle for chemical strange particle abundance deviations from full equilibrium here assumed. We implemented here several features which are more difficult to account for in the full microscopic simulation:

- we incorporate the quantum gas nature for the hadronic particles;

- we treat the entropy contained in the initial thermal state as a parameter and determine it as needed to describe the data;

* Unité associée au CNRS UA 280,

Postal address: LPTHE Université PARIS 7, Tour 24, 5è ét., 2 Place Jussieu, F-75251 CEDEX 05. 
- we explore the consistency of the description with the possible transient presence of a novel form of matter, such as is the locally deconfined quark-gluon plasma (QGP).

The parameters of the thermal model are the temperature $T$ and the chemical potentials $\mu_{i}$ of the different quark flavors. In this discussion it is not necessary to distinguish the $u$ and $d$ flavors and we therefore consider the light quark chemical potential $\mu_{\mathrm{q}}$ and the related fugacity $\lambda_{\mathrm{q}}=\exp \left(\mu_{\mathrm{q}} / T\right)$. Since three quarks together have baryon number one, the baryon number fugacity is $\lambda_{\mathrm{B}}=\lambda_{\mathrm{q}}^{3}$, that is $\mu_{\mathrm{B}}=3 \mu_{\mathrm{q}}$. A finite value of $\mu_{\mathrm{B}}$ indicates that we have a finite baryon density.

We will study in some detail strange particles which contain strange quarks (or antiquarks), associated with strange quark fugacity and chemical potential: $\lambda_{\mathrm{s}}=\exp \left(\mu_{\mathrm{s}} / T\right)$. At relative particle abundance equilibrium the value of $\mu_{\mathrm{s}}$ is fixed within our approach by the requirement that strangeness is conserved. In any hadronic-gas (HG) picture of the reaction leading to high baryon density, there exists typically a considerable asymmetry in the strange and anti-strange particle phase space and hence the requirement of strangeness conservation normally leads to a considerable value of $\mu_{\mathrm{s}}^{\mathrm{HG}}>0$. An exception from this rule exists and is discussed in detail elsewhere [6, 7]. On the other hand, since in the deconfined QGP state both $s$ and $\bar{s}$ quarks are free, one always has $\mu_{\mathrm{s}}^{\mathrm{QGP}}=0$, assuming that strangeness is conserved, $\langle s-\bar{s}\rangle=0$, on the time scale of $\mathrm{A}-\mathrm{A}$ collisions. Said differently, observation of $\mu_{\mathrm{s}} \neq 0$ (that is strange-quark fugacity $\lambda_{\mathrm{s}} \neq 1$ ) strongly suggests that these particles emerge from a final state which is a confined $\mathrm{HG}$ phase.

In the analysis of the collisions of S-ions at $200 \mathrm{GeV}$ A with different nuclear targets carried out at CERN-SPS one finds in the framework of the thermal model [8, 9] that the strange-quark fugacity is $\lambda_{\mathrm{s}} \simeq 1$ (or said alternatively, the strange-quark chemical potential $\mu_{\mathrm{s}} \simeq 0$ ). Furthermore, considering particle production one finds that these collisions are leading to very entropy rich state [10]. Finally, also the strange-particle phase space occupancy is close to equilibrium, $\left(\gamma_{\mathrm{s}} \simeq 1\right)[8,9$ - this suggests the presence of an unusually effective strangeness production mechanisms expected only in the QGP phase. These three results strongly suggest that QGP phase is formed in collisions at $200 \mathrm{GeV} \mathrm{A}$, but considerable additional effort is needed to show this to be the case. Importantly, $\mu_{\mathrm{s}}=0$ is found, despite the expectation that in (a slow) hadronization process of the QGP the memory about the transient, deconfined state would be erased and hadronic particle abundance would reflect conditions of an equilibrated HG. Thus the value $\mu_{\mathrm{s}}=0$ also suggests (if we presume formation of QGP like state) that at CERN the hadronization is sudden and that therefore certain hadronic observables could carry considerable information about the primordial phase. One of our principal results here is a demonstration that as the energy decreases from $\sqrt{s_{\mathrm{NN}}}=8.8$ $\mathrm{GeV}$, at CERN, to $\sqrt{s_{\mathrm{NN}}}=2.5 \mathrm{GeV}$, at AGS, this situation changes with the freeze-out well described by equilibrium HG. This may be considered to indicate that at AGS:

- the QGP phase was not formed at all, or,

- that complete re-equilibration occurs when the primordial QGP hadronizes.

While the freeze-out chemical potentials at CERN energies are well known, the freeze-out temperature remains yet to be determined, despite the relatively large sample of data: the results indicate that the hadronization of the primordial phase is sudden, and one has to permit non-equilibrium abundances of diverse hadronic particles. This introduces additional 
off-equilibrium parameters which need to be determined along with the freeze-out temperature [6]. On the other hand there seems to be no need to introduce non-equilibrium particle abundance parameters (other than strange phase space occupancy factor $\gamma_{\mathbf{s}}$ ) at AGS energies and hence we can determine in this work the freeze-out temperature applicable to AGS we note that the inverse slope parameters of particle spectra, interpreted within the fireball model are the blue shifted freeze-out temperatures $T_{\mathrm{f}}$, the Doppler shift arising from the collective flow phenomena [1].

In this work we will employ state of the art equations of state for both HG and QGP fireballs:

- for HG we use a refined Hagedorn approach which accounts for interactions by treating all hadronic resonances as independent particles. We include numerically the known resonances, considered usually to be Boltzmann particles [6], but in the present work which reaches to lower temperatures $T \leq m_{\pi}$, where $m_{\pi}$ is the pion mass, pions and all mesons are treated as bosons and we also treat nucleons and $\Delta$-resonances as fermions.

- for QGP we take the analytical form of an ideal quark-gluon gas, but amend the number of degrees of freedom to reflect the reduction due to perturbative QCD-interactions, and we introduce non-perturbative thermal mass for quarks and gluons [12].

2. AGS data. We will discuss here $\mathrm{Si}-\mathrm{Au}$ results obtained at $14.6 \mathrm{GeV}$ A by the experiment E802/E859 at BNL, which were presented at the QM'93 meeting [13, 14], in particular:

- the ratio $\mathrm{K}^{-} / \mathrm{K}^{+}=0.22 \pm 0.02$, which is constant in the central rapidity range $1<y<$ 1.6, consistent with the hypothesis that the dominant particle source is a fireball. This ratio varies strongly in target and projectile rapidity regions, as can be expected due to secondary production mechanisms and the presence of baryon-rich reaction fragments;

- the central rapidity ratio $\bar{\Lambda} / \Lambda=(2.0 \pm 0.8) 10^{-3}$ averaged over the acceptance of the spectrometer $(1.1<y<1.7)$.

We will now use these strange particle ratios to determine the values of chemical potentials at freeze-out; we have [6, 77, 8, 9]:

$$
\begin{aligned}
\frac{\bar{\Lambda}}{\Lambda} & =\lambda_{\mathrm{s}}^{-2} \lambda_{\mathrm{q}}^{-4}, \\
\frac{\mathrm{K}^{-}}{\mathrm{K}^{+}} & =\lambda_{\mathrm{s}}^{2} \lambda_{\mathrm{q}}^{-2}
\end{aligned}
$$

We obtain [5]:

$$
\begin{aligned}
& \lambda_{\mathrm{s}}=\frac{\left(\mathrm{K}^{-} / \mathrm{K}^{+}\right)^{1 / 3}}{(\bar{\Lambda} / \Lambda)^{1 / 6}}=1.71\left\{\begin{array}{c}
+0.20 \\
-0.15
\end{array}\right. \\
& \lambda_{\mathrm{q}}=\frac{\left(\mathrm{K}^{+} / \mathrm{K}^{-}\right)^{1 / 6}}{(\bar{\Lambda} / \Lambda)^{1 / 6}}=3.62\left\{\begin{array}{l}
+0.39 \\
-0.26
\end{array}\right.
\end{aligned}
$$


These two results are considerably different from the result obtained at CERN energies: $\lambda_{\mathrm{s}}=1.03 \pm 0.05, \lambda_{\mathrm{q}}=1.49 \pm 0.05$. The AGS result for $\lambda_{\mathrm{s}}$ differs by more than four standard deviations from the CERN value - we note that the high-power roots entering above analysis reduce the significance of the large errors in the data. We further note the relatively high value of $\lambda_{\mathrm{q}}$ which is more than twice as large as that of the CERN experiments and suggestive of considerable baryon density reached at AGS conditions.

An interesting implication and to some extent a verification of the thermal fireball model is that the two particle ratios we consider directly determine the $\bar{p} / p$ ratio - note that Eqs. (11), (2) imply:

$$
\frac{\bar{p}}{p}=\lambda_{\mathrm{q}}^{-6}=\frac{\bar{\Lambda}}{\Lambda} \frac{\mathrm{K}^{-}}{\mathrm{K}^{+}}=(0.44 \pm 0.2) 10^{-3}
$$

We note [13] that at central rapidity $y=1.3$ the $\bar{p}$-yield is given to be $d N / d y \sim 10^{-2}$, which, when combined with the central proton yield $d N / d y \sim 20$, leads to $\bar{p} / p=(0.5 \pm 0.2) 10^{-3}$ (where we estimate the error from the results shown in [13]), which agrees in a remarkable way with the result we obtained above. Since within the error bar the ratio varies by factor two, it is also consistent with the other experimental results reported [15]. We stress that this agreement of the fireball model with experiment may be fortitouse, since the $\bar{p}$ data are still preliminary, while the $p$-yield is strongly influenced by the target spectator tail. We will therefore return to a full discussion of the validity of the fireball model when the $\mathrm{Au}-\mathrm{Au}$ results become available.

3. Theoretical interpretation. A natural question arising here is in how far these values of $\lambda_{\mathrm{s}}, \lambda_{\mathrm{q}}$ are consistent with the expected properties of the hadronic state and what they tell us about other freeze-out conditions. First, let us note a powerful constraints for our analysis: the three thermal parameters $T, \lambda_{\mathrm{s}}, \lambda_{\mathrm{q}}$ are constrained by the requirement that the net strangeness of the source be zero - roughly speaking, the sum of hyperons and anti-kaons must equal the number of kaons, (we of course account for all other strange particles) and the values found in Eqs. (3), (4) are consistent with this constraint. When implementing in detail the strangeness conservation constraint we allow for one off-equilibrium parameter: since strange quark flavor is produced relatively slowly in hadronic interactions (but strangeness produced is easily redistributed among different degrees of freedom), it is customary [8] to weight with a factor $\gamma_{\mathrm{s}}^{n}$ all hadrons containing $n$ strange or anti-strange quarks. This strangeness phase space occupancy factor enters in some theoretical results [6], thus we will allow in the discussion below a range of values: on the low side we take $\gamma_{\mathrm{s}}=0.2$, which is the value typical of $p-p$ collisions, and the upper limit will be $\gamma_{\mathrm{s}}=0.7$, which is found in 200 $\mathrm{GeV}$ A experiments. The equilibrium value is $\gamma_{\mathrm{s}}^{\mathrm{eq}}=1$.

A parameter of all thermal models is the specific entropy (entropy per baryon) content of the source: once the initial thermalized state is established, its evolution to the freeze-out condition is proceeding nearly without entropy production as we have found studying the approach to chemical equilibrium for (isolated) fireballs [16. A most remarkable property of the HG (and also QGP) equations of state of hadronic matter which we use here is that at a given value of specific entropy per baryon (given strangeness conservation constraint) 
corresponds to a nearly constant ratio:

$$
R_{\lambda}=\lambda_{\mathrm{q}} / \lambda_{\mathrm{s}}
$$

In the QGP phase this is well understood [8, 12] - a dimensionless quantity, such as is $S / B$ can only be a function of dimensionless objects, and since $\lambda_{\mathrm{s}}=1$, it is only a function of $\lambda_{\mathrm{q}}$. In $\mathrm{HG}$ this finding which implies a large degree of cancellation, was for us totally unexpected. In Fig. 1 we show for each value of specific entropy $10 \leq S / B \leq 30$ the range of $R_{\lambda}=\lambda_{\mathrm{q}} / \lambda_{\mathrm{s}}$ which is possible when we vary temperature in the wide range $T \in(100,210)$ $\mathrm{MeV}$ and also allow for a wide range in strange particle occupancy $\gamma_{\mathrm{s}} \in(0.2,0.7)$ (lower bars are for $\gamma_{\mathrm{s}}=0.2$ and upper ones for 0.7). We have interpreted in Eq.(2) the kaon ratio in terms of fugacities. This implies $R_{\lambda}=2.12 \pm 0.12$ and we show (right two vertical lines in Fig. (1) that this corresponds to the specific entropy at freeze-out $S / B=13 \pm 1$ (for $\left.\gamma_{\mathrm{s}} \sim 0.4\right)$. This implies after all resonances have decayed a final state ratio of mesons to baryons $R_{\mathrm{m} / \mathrm{b}}=1.6 \pm 0.2$, which is probably compatible with the somewhat lower value 1.2 reported [3]; since in our calculus we account for the enhancement of low $p_{\perp}$ pions, arising from resonance disintegration. In Fig. 11 we also show the value of $R_{\lambda}=\lambda_{\mathrm{q}} / \lambda_{\mathrm{s}} \simeq 1.5$ associated with the $200 \mathrm{GeV}$ A results (left two vertical lines in Fig. 1) — we see that the HG type interpretation at CERN would imply a specific entropy content of $24 \pm 5$, while the high particle flow seen indeed points towards $S / B=40$ more appropriate for a rapidly disintegrating QGP phase [10].

In order to determine the freeze-out temperature $T_{\mathrm{f}}$, we consider (see Fig. 2) the ratio $\bar{\Lambda} / \Lambda$ at fixed $S / B$ as function of $T$. Again we find that there is residual $\gamma_{\mathrm{s}}$ dependence - the thick lines are for the upper limit $\gamma_{\mathrm{s}}=0.7$, the thin lines for the lower limit $\gamma_{\mathrm{s}}=0.2$. The short dashed (upper) lines are for $S / B=14$, the long dashed (lower) ones for $S / B=12$ and the solid lines are for $S / B=13$. The experimental range $\bar{\Lambda} / \Lambda \in(1.2,2.8) 10^{-3}$ thus leads to the range $T_{\mathrm{f}}=127 \pm 8 \mathrm{MeV}$, and we indicate the experimental and theoretical ranges of the values by the error cross in Fig. 2. Given the thermal freeze-out conditions we can obtain the freeze-out baryon density $\rho_{\mathrm{f}}=(0.7 \pm 0.3) \rho_{N}$, where $\rho_{N}=0.16 \mathrm{fm}^{-3}$.

We now turn to the two questions:

1) what are the initial thermal conditions when the fireball is formed?

2) what differences, if any, are there between HG and QGP phases?

In Fig. B we show in the $\mu_{\mathrm{B}}, T$ plane for the HG case (thick lines) and QGP case (thin lines) the different possible histories of the collision. The dashed lines correspond to a fixed energy per baryon $2.55 \mathrm{GeV} \mathrm{A}$ in the $\mathrm{CM}$ frame, while the solid lines are for fixed specific entropy per baryon $S / B=13$. Where the solid and dashed lines meet (within a trapezoidal region determined by one unit error in entropy and an error of $0.15 \mathrm{GeV}$ in $\mathrm{CM}$ energy) we have a consistency between initial energy and final state entropy, thus presumably these are the initial values of thermal parameters. The initial $H G$ state (for $S / B=13, E / B=2.55$ $\mathrm{GeV}$ ) has an energy density $\varepsilon_{0}^{\mathrm{HG}}=2.3 \mathrm{GeV} \mathrm{fm}^{-3}$, and baryon density $\rho_{0}^{\mathrm{HG}}=6 \rho_{N}$. The initial pressure is $P_{0}^{\mathrm{HG}}=0.3 \mathrm{GeV} \mathrm{fm}^{-3}$, which is normally considered a value typical of the deconfined phase. In the QGP phase we find accordingly: $\varepsilon_{0}^{\mathrm{QGP}}=1.2 \mathrm{GeV} \mathrm{fm}^{-3}$, $\rho_{0}^{\mathrm{QGP}}=2.9 \rho_{N}, P_{0}^{\mathrm{QGP}}=0.39 \mathrm{GeV} \mathrm{fm}^{-3}$. Surprizingly, the QGP is the more dilute phase at these conditions, but it can be argued that the greater stiffness of QGP, as compared to HG, reduced the nuclear compression reached in collision. 
Remarkably, we find a very large difference in $\mu_{\mathrm{B}}$ which takes an initial value $\mu_{0}^{\mathrm{HG}}=$ $440 \pm 40 \mathrm{MeV}$ in the $\mathrm{HG}$ scenario and $\mu_{0}^{\mathrm{QGP}}=910 \pm 150 \mathrm{MeV}$ in the QGP case - not shown in the Fig. 3 is that the strangeness conservation requirement leads in the HG to an initial value $\mu_{\mathrm{s}, 0}^{\mathrm{HG}}=0$, just as is in the case of the QGP. We see in Fig. 3 that the initial temperatures $T_{0}$ for QGP and HG scenarios are practically equal. For $E / B=2.55 \pm 0.15$ $\mathrm{GeV}$ and $S / B=13 \pm 1$ we have $T_{0}=190 \pm 30 \mathrm{MeV}$. We recall that the effective temperature seen in the $m_{\perp}$ particle spectra is nearly $T_{0}$ [9, 11]. Thus presence of high inverse slopes in high $m_{\perp}$ spectra with $T \simeq 225 \mathrm{MeV}$ [13] is consistent with our considerations and both equations of state used (HG and QGP). Perhaps a small edge can be given to the QGP case, since one should allow in principle for some entropy production in the transition to HG and for $S / B=11$ one would indeed reach with the central value to $T_{0}^{\mathrm{QGP}} \simeq 215 \mathrm{MeV}$.

The QGP fireball at $S / B=13$ (thin solid line) evolves practically at fixed $\lambda_{\mathrm{q}}=4.8$ and $\lambda_{\mathrm{s}}=1$. For the $\mathrm{HG}$ fireball at fixed $S / B=13$ (thick solid line) there is a strong variation in both these fugacities but as noted in Fig. 2 the ratio $R_{\lambda}^{\mathrm{HG}}\left(=\lambda_{\mathrm{q}} / \lambda_{\mathrm{s}}\right)=2.17$ remains practically constant. The 'experimental' cross is set at $\mu_{\mathrm{B}, \mathrm{f}}=485 \pm 70 \mathrm{MeV}$ and $T_{\mathrm{f}}=127 \pm 8 \mathrm{MeV}$ corresponding to our determination of freeze-out conditions (with $\mu_{\mathrm{s}, \mathrm{f}}=68$ $\mathrm{MeV}$. This appears near the region where the two evolution curves meet (QGP and HG) but in fact both miss each other greatly in a three dimensional display, since the ratio $R_{\lambda}^{\mathrm{QGP}}=\lambda_{\mathrm{q}} / \lambda_{\mathrm{s}}=4.8$ on the QGP path-line and $=2.17$ on the HG path-line.

We have also studied where a possible phase transformation from QGP to HG may be if QGP is produced. Since we are in relative low pressure domain, the pressure of the QGP phase is impacted by the vacuum bag constant $\mathcal{B}$ contribution. We took $\mathcal{B}=0.1 \mathrm{GeV} \mathrm{fm}^{-3}$ (corresponding to $\mathcal{B}^{1 / 4}=170 \mathrm{MeV}$ ). From the resulting phase diagram we obtained that in the event that a first order phase transition is occurring, it would be at $P=0.04 \pm$ $0.01 \mathrm{GeV} \mathrm{fm}^{-3}$ and this determines points on the two evolution paths shown, connected by nearly vertical lines - we note that there is minor re-heating occurring while the baryochemical potential drops by $15 \%$ - however, the major re-equilibration is in the jump from $\lambda_{\mathrm{s}}=1$ in the plasma to $\lambda_{\mathrm{s}} \sim 1.7$ in the HG phase (as discussed above this is still amplified by comparing the greatly different values of $R_{\lambda}$ in both phases). Thus the 'short' connection between the QGP to HG paths would be considerably stretched in full three dimensional display, reflecting on the need to well re-equilibrate the matter in transition. Therefore we believe that there is not a slightest remembrance of the possible QGP history in the AGS data considered here.

However, it is likely that the presence of a primordial phase can be in principle seen in hadronic particle spectra due to the great difference in the $R_{\lambda}$ ratio between QGP and HG cases - the difference is by factor 2.2. Thus e.g. the ratio of $\mathrm{K}^{+}$to $\pi^{+}$particle abundance at fixed $m_{\perp}$ should increase at large $m_{\perp}$ supposing that the high $m_{\perp}$ particles are directly emitted from the possibly formed QGP phase. Since in the fireball model

$$
\left.\frac{\mathrm{K}^{+}}{\pi^{+}}\right|_{m_{\perp}}=R_{\lambda}
$$

we should see that the high $m_{\perp}$ ratio decreases just by factor 2.2 as the high $m_{\perp}$ QGP primordial contribution are overcome by hadronization yields, and at still smaller $m_{\perp}$ by even more as the pion abundance is diluted by the $\Delta$ decays (an effect which we can fully 
account for given the model for the freeze-out conditions we developed).

4. Conclusions and outlook. We have reached a full and satisfactory description of the freeze-out conditions at AGS energies, employing thermal fireball model. We have alluded to a number of consistency checks, e.g. have mentioned that the meson to baryon ratio in the final state $R_{\mathrm{m} / \mathrm{b}}=1.6 \pm 0.2$, and also noted that the ratio $\bar{p} / p=0.510^{-3}$ is of the correct magnitude. We note that given the freeze-out conditions we can compute the abundances of most hadronic particles in the final state, and we intend to develop a more detailed and comprehensive analysis once more precise input data become available.

Should QGP be formed at these low energies, we hope that akin to the situation at $200 \mathrm{GeV}$ A, one will be able to see considerable anomalies in abundances of multi-strange anti-baryons. These are produced extremely rarely in the HG environment and normally one would not believe that they can be observed for beam energies at about 14.6 GeV A. However, should at AGS indeed the QGP phase be formed, their abundance would be only little reduced as compared to the $200 \mathrm{GeV}$ A situation, while the experimental sensitivity would be greatly amplified, since the entropy content and hence the final state multiplicity is much smaller at these lower energies. Given the theoretical results we have presented regarding the initial thermal conditions reached in the central fireball in $14.6 \mathrm{GeV}$ A collisions, it is hard not to envision QGP formation at AGS and hence we would like to encourage strongly that a search be made for these hadronic observables [17] of the QGP phase. Thus we are in particular interested in central rapidity data about (multi-)strange anti-baryons which we hope could help resolve the question if there has been formation of transient deconfined phase. In particular we may be able to determine the value of $\gamma_{\mathbf{s}}$; we need to look at the ratios:

$$
R_{\overline{\mathrm{s}}}=\gamma_{\mathrm{s}} \frac{\lambda_{\mathrm{q}}}{\lambda_{\mathrm{s}}}=\left.\left.\left.\gamma_{\mathrm{s}} R_{\lambda} \sim \frac{1}{2} \frac{\bar{\Lambda}+\overline{\Sigma^{0}}}{\bar{p}}\right|_{m_{\perp}} \sim 2 \frac{\overline{\Xi^{-}}}{\bar{\Lambda}+\overline{\Sigma^{0}}}\right|_{m_{\perp}} \sim \frac{1}{2} \frac{\overline{\Omega^{-}}}{\overline{\Xi^{-}}}\right|_{m_{\perp}} .
$$

Resonance decays influence these yields, and hence the above relation holds approximately, but increasingly exactly as the strangeness content increases. If QGP is formed and the strangeness phase space were fully saturated, we would expect that the $R_{\overline{\mathrm{s}}}$ ratio is indeed greater than unity. Due to the large value of $\lambda_{\mathrm{q}}$ we found, if QGP is formed with $\gamma_{\mathrm{s}} \simeq 0.7$ and $R_{\lambda}=\lambda_{\mathrm{q}} / \lambda_{\mathrm{s}}=4.8$ governing emission at high $m_{\perp}$, we obtain $R_{\overline{\mathrm{s}}} \simeq 3$. Using the $\mathrm{HG}$ values $\gamma_{\mathrm{s}} \sim 0.15, R_{\lambda}=2.1$ one finds a yield typical for $p$ - $p$ reactions, with $R_{\overline{\mathrm{s}}} \sim 0.3$. We recall here that for the CERN experiment the corresponding discussion addressed the ratio of yields of $\bar{\Xi} / \bar{\Lambda}$ and the high value of $\gamma_{\mathrm{s}} \sim 0.7$ found there arises from the remarkable result that $R_{\overline{\mathrm{s}}}$ is $\mathcal{O}(1)$ at $200 \mathrm{GeV}$.

Even though we have here discussed only the Si beam experiments at $14.6 \mathrm{GeV} \mathrm{A}$ involving collisions with the heaviest target $(\mathrm{Au})$ as is appropriate for the here considered fireball model, our analysis can be easily continued to the case of $\mathrm{Au}-\mathrm{Au}$ collisions at 11-12 $\mathrm{GeV}$ A [3, 18]. Because of the projectile-target symmetry the CM specific energy (assuming full stopping or equal baryon and energy stopping) is for the generally considered value of 11.7 GeV A exactly the value we assumed here, namely $\sqrt{s_{\mathrm{NN}}}=2.55 \mathrm{GeV}$. We note that a slight increase in the ratio of $\mathrm{K}^{+} / \mathrm{K}^{-}$from 4.5 to perhaps 5 would imply a slight drop in the specific entropy (see Eq. (2) and Fig. 1), perhaps by 1/2 unit. This causes only incremental 
changes in all freeze-out results and thus there will be little change in final state particle abundances. However, we find, within the framework of HG model, a considerable sensitivity of the initial conditions (baryon density, pressure and energy density), seen as function of the entropy content: a drop by one unit of $S / B$ from 13 to 12 implies doubling of all these initial values. Thus it is well possible that much more extreme conditions are reached in the $\mathrm{Au}-\mathrm{Au}$ collisions as compared to $\mathrm{Si}-\mathrm{Au}$, even if the reaction products and multiplicity appear to be similar for both systems.

In summary: we have used that results on strange particle ratios $\bar{\Lambda} / \Lambda$ and $\mathrm{K}^{-} / \mathrm{K}^{+}$from AGS in a fireball model framework and have determined freeze-out values of temperature $\left(T_{\mathrm{f}}=127 \pm 8 \mathrm{MeV}\right)$, and chemical potentials $\left(\mu_{\mathrm{B}, \mathrm{f}}=485 \pm 70 \mathrm{MeV}\right.$ and $\mu_{\mathrm{s}, \mathrm{f}}=68(+20,-16)$ $\mathrm{MeV}$, final state specific entropy $S / B=13 \pm 1$ accompanied by meson to baryon particle ratio $R_{\mathrm{m} / \mathrm{b}}=1.6 \pm 0.2$, and also within the HG and QGP models, the (extreme) initial conditions prevailing when the fireball is formed, in particular $T_{0}=190 \pm 30 \mathrm{MeV}$.

Acknowledgements. Work of JR is in part supported by US-Department of Energy under grant DE-FG02-92ER40733. JR thanks his colleagues for their kind hospitality in Paris.

\section{References}

[1] E. Quercigh, in Particle Production in Highly Excited Matter; Plenum Press (New York, 1993), NATO ASI Series B303, 499 (1993), Eds. H.H. Gutbrod and J. Rafelski.

[2] Y. Pang, T.J. Schlagel and S.H. Kahana, Phys. Rev. Lett. 68 (1992) 2743;

T.J. Schlagel, S.H. Kahana and Y. Pang, Phys. Rev. Lett. 69 (1992) 1290

S. H. Kahana, Y. Pang and T. J. Schlagel, Physics at the AGS with a Relativistic Cascade, BNL preprint 48888, February 1993;

[3] M. Gonin, lecture at Orsay, 31 January 1994, (IPN-Orsay report, February 1994).

[4] M. Gaździcki and NA35 collaboration, private communication and to be published.

[5] J. Rafelski and M. Danos, "Strangeness Flow Difference in Nuclear Collisions at 15 and 200 GeV A", submitted to Phys. Rev. Lett. NIST preprint 1993.

[6] J. Letessier, A. Tounsi, and J. Rafelski, Phys. Lett. B292 (1992) 417.

J. Letessier, J. Rafelski and A. Tounsi, Phys. Lett. B321 (1994) 394.

[7] J. Cleymans and H. Satz, Z. Phys. C57, 135 (1993).

J. Cleymans, K. Redlich, H. Satz and E. Suhonen, Z. Phys. C58 (1993) 347.

[8] J. Rafelski, Phys. Lett. B262 (1991) 333; and Nucl. Phys. A544 (1992) 279c.

[9] J. Letessier, A. Tounsi, U. Heinz, J. Sollfrank, and J. Rafelski, Paris preprint PAR/LPTHE/92-27R, submitted to Phys. Rev. D.

J. Sollfrank, M. Gaździcki, U. Heinz, and J. Rafelski, Chemical Freeze-out Conditions in Central S-S Collisions at 200 A GeV, Z. Phys. C (1994) in press. 
[10] J. Letessier, A. Tounsi, U. Heinz, J. Sollfrank and J.Rafelski, Phys. Rev. Lett. 70 (1993) 353.

[11] K. S. Lee, U. Heinz, and E. Schnedermann, Z. Phys. C48 (1990) 525; E. Schnedermann and U. Heinz, Phys. Rev. Lett. 69 (1992) 2908; E. Schnedermann, J. Sollfrank, and U. Heinz, in Particle Production in Highly Excited Matter; Plenum Press (New York, 1993), NATO ASI Series B303 (1993) 175, Eds. H.H. Gutbrod and J. Rafelski.

[12] J. Letessier, J. Rafelski and A. Tounsi, Phys. Lett. B323 (1994) 393.

[13] G.S.F. Stephans et al. E859 collaboration, Nuc. Phys. A566 (1994) 269c.

[14] D.P. Morrison et al. E859 collaboration, Nuc. Phys. A566 (1994) 457c.

[15] J. Barrette et. al., E814 Collaboration, Phys. Rev. Lett. 70 (1993) 1763.

[16] J. Letessier, J. Rafelski and A. Tounsi, Gluon Production, Cooling and Entropy in Nuclear Collisions, Paris preprint LPTHE/93-51, October 1993, submitted to Phys. Rev. Lett.

[17] J. Rafelski, Phys. Reports 88 (1982) 331; See also [8] and references therein.

[18] M. Gonin et al. E802/E866 collaboration, Nuc. Phys. A566 (1994) 601c.

\section{FIGURE CAPTIONS}

Figure 1: For a given specific entropy per baryon $S / B$ we show the range of allowable values $\lambda_{\mathrm{q}} / \lambda_{\mathrm{s}}$ values in HG phase, when temperature is varied between 100 and $210 \mathrm{MeV}$ and the strangeness occupancy factor $\gamma_{\mathrm{s}}$ between is 0.2 (lower values) and 0.7 (upper values). Strangeness conservation constraint is imposed. See text for discussion of vertical lines.

Figure 2: The ratio $\bar{\Lambda} / \Lambda$ as function of temperature, at fixed specific entropy and for $\gamma_{\mathrm{s}}=0.7$ (thick lines) and 0.2 (thin lines). Solid lines $S / B=13$, short dashed $S / B=14$, long dashed $S / B=12$.

Figure 3: $\quad \mu_{\mathrm{B}}-T$ plane with thick lines: HG model; thin lines: QGP model. Dashed lines: fixed energy per baryon $E / B=2.55 \mathrm{GeV}$, upper for HG, lower for QGP. Solid lines: fixed specific entropy $S / B=13$, upper for QGP, lower for HG model. Trapezoidal regions enclose the initial condition (see text). Solid line connecting QGP and HG with dotted lines left/right: possible phase transition at $P=0.04 \pm 0.01 \mathrm{GeV} / \mathrm{fm}^{3}$. Note that continuations of $S / B=13$ lines beyond transition or hadronization points are dotted. 


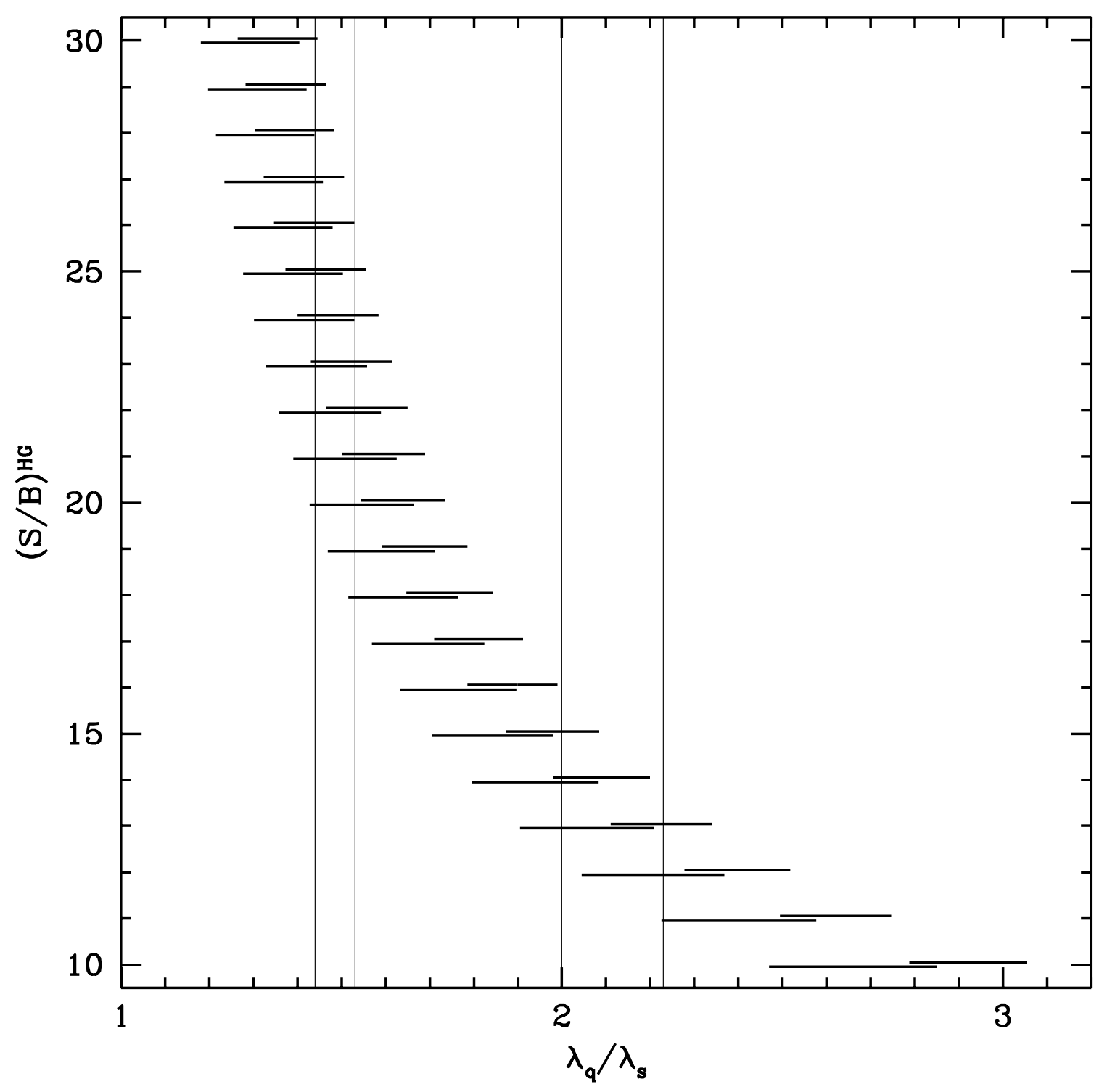

FIGURE 1 
Strange Particle Freeze-Out at AGS

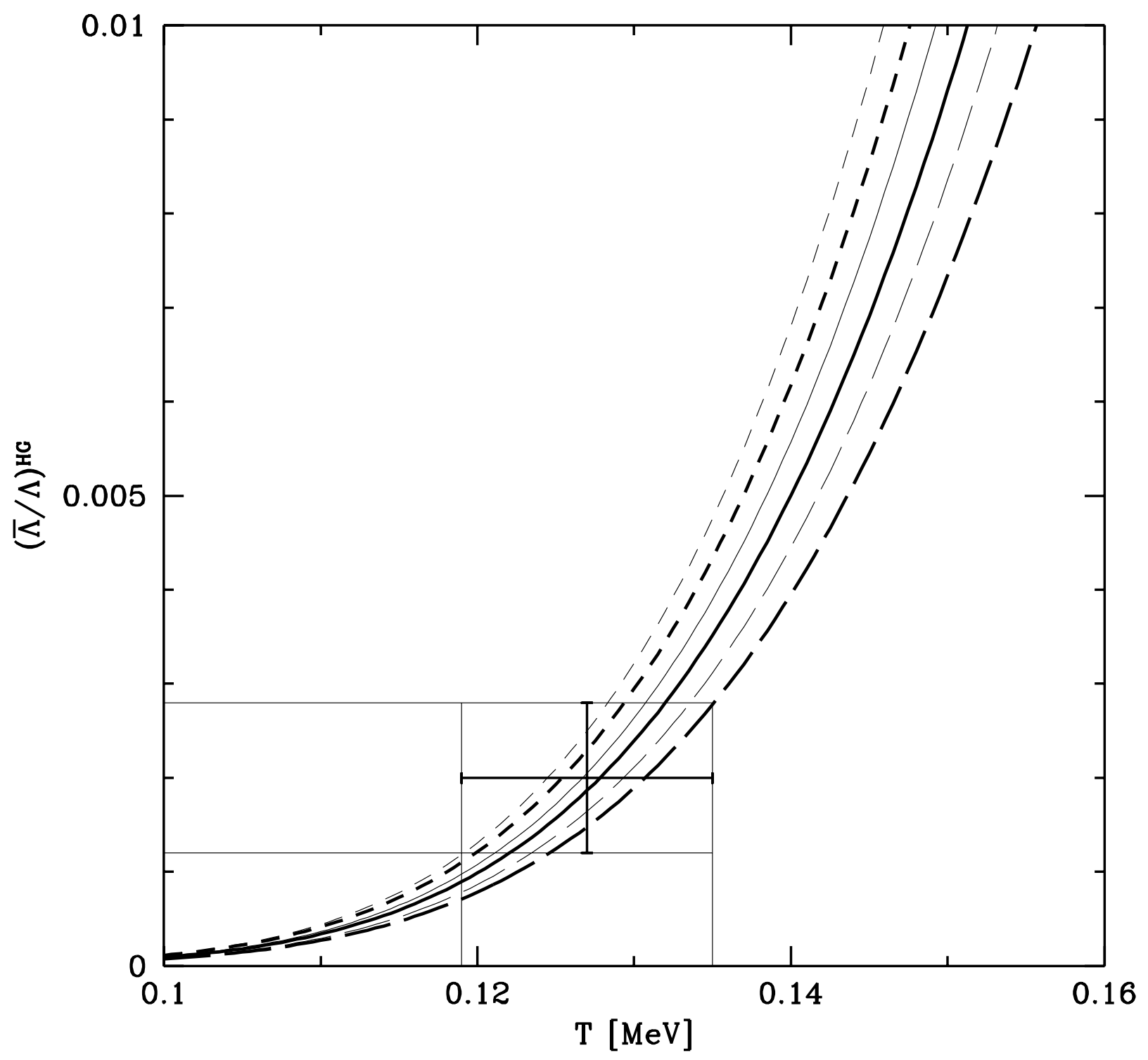

FIGURE 2 


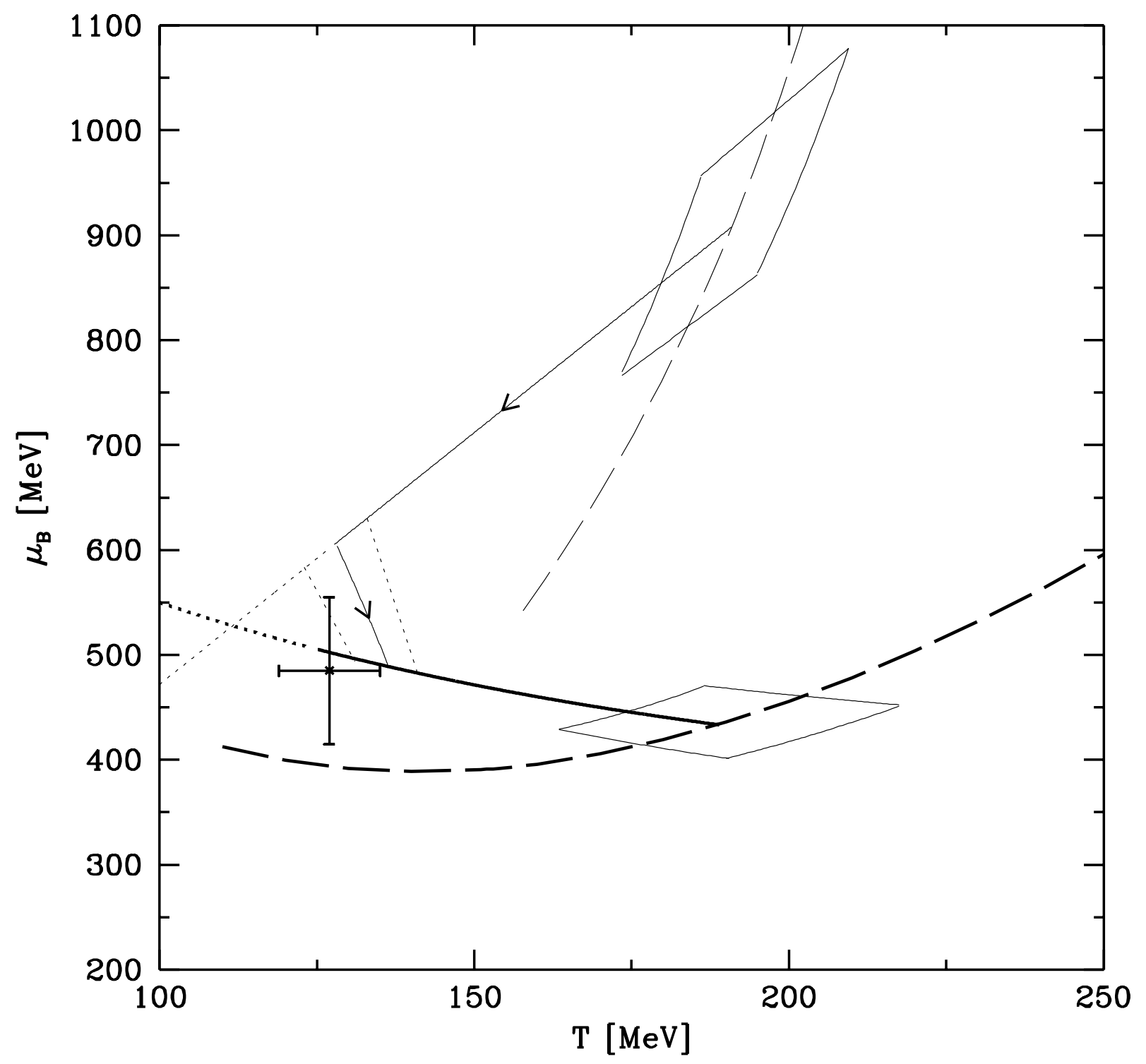

FIGURE 3 